\title{
Collaborating filtering using unsupervised learning for image reconstruction from missing data
}

\author{
Oumayma Banouar ${ }^{1,2^{*}}$ (D), Souad Mohaoui ${ }^{1}$ and Said Raghay ${ }^{1}$
}

\begin{abstract}
In the image acquisition process, important information in an image can be lost due to noise, occlusion, or even faulty image sensors. Therefore, we often have images with missing and/or corrupted pixels. In this work, we address the problem of image completion using a matrix completion approach that minimizes the nuclear norm to recover missing pixels in the image. The image matrix has a low rank. The proposed approach uses the nuclear norm function as a surrogate of the rank function in the aim to resolve the problem of rank minimization that is known as an NP-hard problem. It is an adaptation of the collaborating filtering approach used for users' profile construction. The main advantage of this approach is that it uses a learning process to classify pixels into clusters and exploits them to run a predictive method in the aim to recover the missing or unknown data. For performance evaluation, the proposed approach and the existing matrix completion methods are compared for image reconstruction according to the PSNR measure. These methods are applied on a dataset composed of standard images used for image processing. All the recovered images obtained during experimentation are also dressed to compare them visually. Simulation results verify that the proposed approach achieves better performances than the existing matrix completion methods used for image reconstruction from missing data.
\end{abstract}

Keywords: Image reconstruction, Bi-clustering, Matrix completion, Unsupervised learning, Prediction, Rank function, Nuclear norm function, Surrogate model

\section{Introduction}

The reconstruction of missing pixels from an incomplete image is a very active research area in image processing. A simple model for such problem can be defined as follows: given an image which is incomplete, i.e., it has missing pixels, the purpose is to fill its missing pixels based on some observed pixels. In analogy with matrix completion problem, the problem of recovering missing pixels in an image can be referred to as image completion problem.

In this work, we are interested in recovering missing pixels from an incomplete image using a matrix completion method based on the minimization of the nuclear norm of a matrix. The nuclear norm minimization is a category of low-rank matrix approximation methods. Mathematically

\section{*Correspondence: o.banouar@edu.uca.ma}

'Department of Applied Mathematics and Computer Science, Faculty of Science and Technics, Cadi Ayyad University, Marrakesh, Morocco

${ }^{2}$ Higher School of Technology, Sidi Mohamed Ben Abdellah University, Fez, Morocco speaking, given an incomplete image $X$, missing values are estimated given observed pixels: $\left\{D_{i j} / i, j \in \Omega\right\}$ where $\Omega$ denotes the set of observed entries. The common assumption is that the matrix should be low-rank (most images have low rank). Then, a direct approach is to minimize the rank of the matrix with certain constraints. This problem is NP-hard; a convex relaxation is often used to make the minimization tractable. As the rank function is simply the number of nonvanishing singular values, the most appropriate choice is to replace the rank function with the nuclear norm. Therefore, the proposed approach is based on the nuclear norm minimization that is the surrogate model of the rank minimization.

The approach used in this work is proposed in [1] for users' profile construction. It uses a matrix completion method based on nuclear norm optimization of the matrix to predict users' preferences about items. A bi-clustering process is adopted to detect users' clusters and items' clusters in the aim to promote the personal relevancy 
concept [2]. It applies the prediction process on the ratings given by users that share almost the same preferences.

The main problem with recovering missing data in images is the sparcity of the matrix that modelize them. With the same principle, we adapt the users' profile construction method to recover the missing pixels. The obtained experimental results proved the efficiency of the proposed prediction process. The proposed approach is applied on a benchmark that contains standard images for image processing. They are gray-level images that have different histograms. The obtained results are compared visually to those obtained by applying different nuclear norm optimization algorithms. The peak signal-to-noise ratio (PSNR) measure is also calculated for each recovered image.

The remaining of this article is organized as the follows. Section 2 presents the role of nuclear norm minimization in the optimization of low-rank matrices. It exposes then the problem statement and explains the proposed approach. It also reviews the related works. Section 3 addresses the experimental protocol and discusses the obtained results. The conclusion closes the paper.

\section{Methods}

\subsection{Minimization of low-rank matrices using nuclear norm minimization}

In the area of engineering and applied science such as machine learning and computer vision, a wide range of problems can be or have been represented under low-rank minimization framework, since the low-rank formulation seems to be able to capture the low-order structure of the underlying problems.

In many practical problems, one would like to guess the missing entries of an $n_{1} \times n_{2}$ matrix from a sampling $\Omega$ of its entries. This problem is known as the matrix completion problem. It comes up in a great number of applications including those of collaborating filtering. The collaborating filtering is the task of automatic predicting of the entries in an unknown data matrix. A popular example is the movie recommendation case where the task is to make automatic predictions about the interests of a user by collecting taste information from its formal interests or by collecting them from other users.

In mathematical terms, this problem is posed as follows:

A data matrix $X \in \mathbb{R}^{n_{1} \times n_{2}}$ is the matrix to be known as much as possible. The only information available about it is a sampling set of entries $M_{i j},(i, j) \in \Omega$, where $\Omega$ is a subset of the complete set of entries $\left\{1, \ldots, n_{1}\right\} \times\left\{1, \ldots, n_{2}\right\}$.

Very few factors contribute to an individual's tastes. Therefore, the problem of matrix completion is an optimization problem of a low-rank $r$ matrix from a sample of its entries. The matrix rank satisfies $r \leq \min \left(n_{1}, n_{2}\right)$. Such a matrix is represented by counting $n_{1} \times n_{2}$ numbers but has only $r \times\left(n_{1} \times n_{2}-r\right)$ degrees of freedom. When the matrix rank is small and its dimension is large, then the data matrix carries much less information than its ambient dimension suggests. In the case of collaborative prediction movie recommendation system, users-rows of the matrix-are given the opportunity to rate items-columns of the data matrix. However, they usually rate very few ones so there are very few scattered observed entries of this data matrix. In this case, the users-ratings matrix is approximately low-rank, because as mentioned, it is commonly believed that only very few factors contribute to an individual's tastes or preferences. These preferences are stored in a user profile [1]. In the same analogy, matrix completion can be used to restore images with missing data. From limited information, we aim to recover the image, i.e., infer the many missing pixels.

\subsection{Problem statement}

Given $\Omega \subset\left[n_{1}\right] \times\left[n_{2}\right]$ a set of elements of an unknown rank-r matrix, $X \in \mathbb{R}^{n_{1} \times n_{2}}$. The values of elements $M_{i j},(i, j) \in \Omega$ are known. The task is to recover incomplete matrix $X$. Formally, the low-rank matrix completion problem is given by:

$$
\begin{cases}\text { minimize } & \operatorname{rank}(X) \\ \text { subject to } & P_{\Omega}(X)=P_{\Omega}(M)\end{cases}
$$

where $P_{\Omega}: \mathbb{R}^{n_{1} \times n_{2}} \longrightarrow \mathbb{R}^{n_{1} \times n_{2}}$ is the orthogonal projection onto the subspace of matrices that vanish outside of $\Omega,(i, j) \in \Omega$ if and only if $M_{i j}$ is observed. $P_{\Omega}(X)$ is defined by:

$$
P_{\Omega}(X)= \begin{cases}X_{i j} & \text { if }(i, j) \in \Omega \\ 0 & \text { otherwise }\end{cases}
$$

The data known in $M$ is given by $P_{\Omega}(M)$. The matrix $X$ is recovered then from $P_{\Omega}(X)$ if it is the unique matrix of rank less or equal to $r$ and consistent with the data.

In a practical point of view, the rank minimization problem is an NP-hard problem. Algorithms are not capable to resolve it in time once the matrices have an important dimension. They require time doubly exponential in the dimension of the matrix to find the exact solution. Authors in [3] proposed the nuclear norm minimization method. Replacing the rank of a matrix by its nuclear norm can be justified as a convex relaxation (the nuclear norm $\|X\|_{*}=\sum_{i} \sigma_{i}(X)$ is the largest convex lower bound of $\operatorname{rank}(X)$ on the ball $\left\{X /\|X\|_{*}=\sigma(X) \leq 1\right\}$ [3]). Consequently, the problem (2) is then replaced by the following:

$$
\begin{cases}\operatorname{minimize} v & \|X\|_{*} \\ \text { subject to } & P_{\Omega}(X)=P_{\Omega}(M)\end{cases}
$$


where the nuclear norm $\|X\|_{*}$ is defined as the sum of its singular values: $\|X\|_{*}=\sum_{i} \sigma_{i}(X)$.

Since the nuclear norm ball $\left\{X:\|X\|_{*} \leq 1\right\}$ is the convex hull of the set of rank-one matrices with spectral norm bounded by one, authors in [4] interpret that under suitable conditions, the rank minimization program (2) and the convex program (3) are formally equivalent in the sense that they have exactly the same unique solution.

Matrix completion problem is not as ill posed as thought. It is possible to resolve it by convex programming. The rank function counts the number of nonvanishing singular values when the nuclear norm sums their amplitude. The nuclear norm is a convex function. It can be optimized efficiently via semidefinite programming.

The following theorem is demonstrated by authors in [4].

Theorem 1 Let $M$ be an $n_{1} \times n_{2}$ matrix of a rank $r$ sampled from the random orthogonal model, and put $n=\max \left(n_{1}, n_{2}\right)$. Suppose we observe $m$ entries of $M$ with locations sampled uniformly at random. Then, they are numerical constants $C$ and $c$ such that if:

$$
m \geq C n^{5 / 4} r \log n
$$

The minimizer to the problem (3) is unique and equal to $M$ with probability at least $1-\mathrm{Cn}^{-3}$; that is to say, the semidefinite program (3) recovers all the entries of $M$ with no error. In addition, if $r \leq n^{1 / 5}$, then the recovery is exact with probability at least $1-\mathrm{Cn}^{-3}$ provided that:

$$
m \geq C n^{(6 / 5)} r \log n
$$

Under the hypothesis of Theorem 1, there is a unique low-rank matrix, which is consistent with the observed entries. This matrix can be recovered by the convex optimization (3). For most problems, the nuclear norm relaxation is formally equivalent to the combinatorial hard rank minimization problem.

If the coherence is low, few samples are required to recover $M$. As an example, matrices with incoherent column and row space matrices with random orthogonal model or those with small components of the singular vectors of $M$.

Conventional semidefinite programming solvers such as SDPT3 [5] and SeDeMi [6] solve the problem (3). However, such solvers are usually based on interior-point methods and cannot deal with large matrices. They can only solve problems of size at most hundreds by hundreds on a moderate computer. These solvers are problematic when the size of the matrix is large. They need to solve huge systems of linear equations to compute the Newton direction. To be precise, SDTP handles only square matrices with the size less than 100. Another alternative is to think of using iterative solvers such as the method of conjugate gradients to solve the Newton system. However, it is still problematic as well since it is well known that the condition number of the Newton system increases rapidly as one gets closer to the solution. Furthermore, none of these general-purpose solvers use the fact that the solution may have low rank.

Therefore, the first-order methods are used to complete large low-rank matrices by solving (3).

In the special matrix completion setting presented in (3), $P_{\Omega}(X)$ is the orthogonal projector onto the span of matrices vanishing outside of $\Omega$. Therefore, the $(i, j)$ th component of $P_{\Omega}(X)$ is equal to $X_{i j}$ if $(i, j) \in$ $\Omega$ and 0 otherwise. $X \in \mathbb{R}^{n_{1} \times n_{2}}$ is then the optimization variable. Fix $\tau>0$ and a sequence $\delta_{k}$ of scalar step sizes. Starting with $Y_{0}=0\left(\in \mathbb{R}^{n_{1} \times n_{2}}\right)$, the algorithm defines until a stopping criterion is reached:

$$
\left\{\begin{array}{l}
X_{k}=\operatorname{shrink}\left(Y_{k-1}, \tau\right) \\
Y_{k}=Y_{k+1}+\delta_{k} P_{\Omega}\left(M-X_{k}\right)
\end{array}\right.
$$

$\operatorname{shrink}(x, \lambda)$ is a nonlinear function that applies a softthresholding rule at level $\lambda$ to the singular values of the input matrix. The key property here is that for large values of $\tau$, the sequence $X_{k}$ converges to a solution which very nearly minimizes (3). Hence, at each step, one only needs to compute at most one singular value decomposition and perform a few elementary matrix additions.

\subsection{The singular value thresholding algorithm}

The most popular approaches to matrix completion in literature are the thresholding methods that can be divided into two groups: one-step thresholding methods and iterative thresholding methods. Despite the strong theoretical guaranties which have been obtained for one-step thresholding procedures, they show poor behavior in practice and only work under the uniform sampling distribution which is not realistic in many practical situations [7]. On the other hand, iterative thresholding methods are well adapted for general nonuniform distribution as well as they show practical performances as in [4]. Authors in [8] proposed a first-order singular value thresholding algorithm SVT which is a key subroutine in many numerical schemes for solving nuclear norm minimization. The conventional approach for SVT is to find the singular value decomposition SVD of the matrix, then to shrink its singular values.

\section{The singular value decomposition step}

The singular value shrinkage operator is the key building block of the SVT algorithm. Consider the singular value decomposition SVD of a matrix $X \in \mathbb{R}^{n_{1} \times n_{2}}$ of rank $r$

$$
X=U \Sigma V^{*} \quad \text { Where } \quad \Sigma=\operatorname{diag}\left(\left\{\sigma_{i}\right\}_{1 \leq i \leq \tau}\right)
$$

where $U$ and $V$ are respectively $n_{1} \times r$ and $n_{2} \times r$ matrices with orthonormal columns, and the singular values $\sigma_{i}$ are 
positive. For each $\tau \geq 0$, the soft-thresholding operator $D_{\tau}$ is defined as follows:

$$
D_{\tau}(X)=U D_{\tau}(\Sigma) V^{*}=U .\left(\begin{array}{lll}
\left(\sigma_{1}-\tau\right)_{+} & & \\
& \ddots & \\
& & \left(\sigma_{r}-\tau\right)_{+}
\end{array}\right) \cdot V^{*}
$$

where $t_{+}$is the positive part of $t$ defined by:

$$
\left(\sigma_{i}-\tau\right)_{+}=\max \left(0, \sigma_{i}-\tau\right)= \begin{cases}\sigma_{i}-\tau & \text { if } \sigma_{i}-\tau>0 \\ 0 & \text { otherwise }\end{cases}
$$

In other words, in $D_{\tau}(X)$, the singular vectors of $X$ are kept and the singular values are shrinked by the softthresholding.

Even though the SVD may not be unique, it is easy to see that the singular value shrinkage operators are well defined. In some sense, this shrinkage operator is a straightforward extension of the soft-thresholding rule for scalars and vectors. In particular, note that if many of the singular values of $X$ are below the threshold $\tau$, the rank of $D_{\tau}$ may be considerably lower than that of $X$, just like the soft-thresholding rule applied to vectors leads to sparser outputs whenever some entries of the input are below threshold.

The singular value thresholding operator is the proximal operator associated with the nuclear norm. The proximal operator has its origins in convex optimization theory, and it has been widely used for non-smooth convex optimization problems, such as the $l_{1}$-norm minimization problems arising from compressed sensing [9] and related areas. It is well known that the proximal operator of the $l_{1}$-norm is the soft-thresholding operator, and soft-thresholding-based algorithms are proposed to solve $l_{1}$-norm minimization problems [10].

\section{Shrinkage iteration step}

The singular value thresholding SVT algorithm approximates the minimization (3) by:

$$
\left\{\begin{array}{l}
\min _{X} \tau\|X\|_{*}+\frac{1}{2}\|X\|_{F}^{2} \\
\text { subject to } X_{i j}=M_{i j}
\end{array}\right.
$$

with a large parameter $\tau$. $\|\cdot\|_{F}$ denotes the matrix Frobenius norm or the square root of the summation of squares of all entries. Then, it applies a gradient ascent algorithm to its dual problem. The iteration is:

$$
\left\{\begin{array}{l}
X_{k}=D_{\tau}\left(Y_{k-1}, \tau\right) \\
Y_{k}=Y_{k+1}+\delta_{k} P_{\Omega}\left(M-X_{k}\right)
\end{array}\right.
$$

where $D_{\tau}$ is the SVT operator defined as:

$$
D_{\tau}=\arg \min \frac{1}{2}\|Y-X\|_{F}+\tau\|X\|_{*}, X \in \mathbb{R}^{n_{1} \times n_{2}}
$$

The iteration is called the SVT algorithm, and it was shown in [11] to be an efficient algorithm for huge lowrank matrix completion. Two crucial properties make the SVT algorithm suitable for matrix completion.

- Low-rank property: The matrices $X_{k}$ turn out to have low rank, and hence, the algorithm has minimum storage requirement since it only needs to keep principal factors in memory.

- Sparsity: For each $k \geq 0, Y_{k}$ vanishes outside of $\Omega$ and is, therefore, sparse, a fact, which can be used to evaluate the shrink function rapidly.

\section{The SVT algorithm}

The initial step of the SVT algorithm is to start with the following:

- $Y_{0}=0$;

- Choosing a large $\tau$ to make sure that the solution of (11) is close enough to the solution of (3).

- Defining $k_{0}$ as the integer that obeys to: $\frac{\tau}{\delta\left\|P_{\Omega}(M)\right\|} \in\left(k_{0}-1, k_{0}\right)$

- Since $Y_{0}=0, X_{k}=0, Y_{k}=k \delta P_{\Omega}(M), k=1, \ldots, k_{0}$

The stopping criteria of the SVT algorithm is motivated by the first-order optimality conditions for the minimization of the problem (10). The solution $X_{\tau}^{*}$ to (11) must verify:

$$
\left\{\begin{array}{l}
X=D_{\tau}(Y) \\
P_{\Omega}(X-M)=0
\end{array}\right.
$$

where $Y$ is a matrix vanishing outside of $\Omega^{c}$. Therefore, to make sure that $X_{k}$ is close to $X_{\tau}^{*}$, it is sufficient to check how close $\left(X_{k}, Y_{k-1}\right)$ is obeying (13). By definition, the first equation in (13) is always true. Therefore, it is natural to stop (12) when the error in the second equation is below a specified tolerance:

$$
\frac{\left\|P_{\Omega}(X-M)\right\|_{F}}{\left\|P_{\Omega}(M)\right\|_{F}} \leq \epsilon
$$

The matrix completion problem can be viewed as a special case of the matrix recovery matrix, where one has to recover the missing entries of a matrix, given limited number of known entries.

\subsection{Literature review}

Other works dressed other algorithms in the attempt to minimize the nuclear norm of low-rank sparse matrix. Authors in [12] presented the fixed-point continuation (FPC) algorithm. It combines the fixed-point continuation [13] with Bregman iteration [14]. The iteration is as follows: 


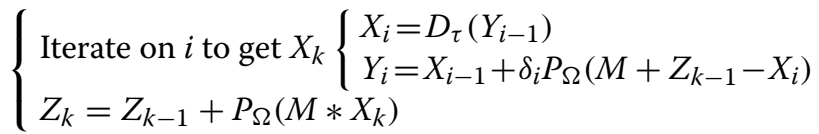

In fact, the FPC algorithm is a gradient ascent algorithm applied to an augmented Lagrangian of (3). The augmented Lagrangian multiplier (ALM) method in [15] reformulates the problem into:

$$
\left\{\begin{array}{l}
\min _{X}\|X\|_{*} \\
\text { subject to } X+E=P_{\Omega}(M), P_{\Omega}(E)=0,
\end{array}\right.
$$

where $E$ is an auxiliary variable. The corresponding (partial) ALM function is:

$$
\begin{aligned}
\Gamma(X, E, Y, \mu)= & \|X\|_{*}+<Y, P_{\Omega}(M)-X-E> \\
& +\frac{\mu}{2}\left\|P_{\Omega}(M)-X-E\right\|_{F}^{2} \text { with } P_{\Omega}(E)=0
\end{aligned}
$$

An inexact gradient ascent is applied to the ALM and leads to the following algorithm:

$$
\left\{\begin{array}{l}
X_{k}=D_{\mu_{k}^{-1}}\left(P_{\Omega}(M)-E_{k}+\mu_{k}^{-1} Y_{k-1}\right) \\
E_{k}=P_{\Omega^{c}}\left(X_{k}\right) \\
Y_{k}=Y_{k-1}+\mu_{k} P_{\Omega}\left(M-X_{k}\right)
\end{array}\right.
$$

For all these algorithms, the SVT operator is the key to make them converge to low-rank matrices.

Just like the FPC and SVT algorithms, the proximal gradient (PG) [16] algorithm for matrix completion needs to compute the SVD at each iteration. It is as simple as the cited algorithms.

There are two main advantages of the SVT algorithm over the FPC and the PG algorithms when the former is applied to solve the problem of matrix completion.
First, in some cases, we dispose a sequence of lowrank iterates; in contrast, so many iterates at the initial phase of the FPC or PG algorithms may not have low rank even though the optimal solution itself has low rank. We observed this behavior when we applied them to solve the problem of matrix completion.

Second, the intermediate matrices generated during the resolution of our problem are sparse due to the sparcity of $\Omega$, the set of observation. This makes the SVT algorithm computationally more attractive. Indeed, the generated matrices by FPC and PG algorithms may not be sparse and specially for the last one.

The first-order methods presented above are the basis of a number of recent works that minimize the nuclear norm of a matrix to recover an image with missing data.

In [17], authors proposed a two-step proximal gradient algorithm to solve nuclear norm regularized least squares for the purpose of recovering low-rank data matrix from sampling of its entries. Each iteration generated by the proposed algorithm is a combination of the latest three points, namely, the previous point, the current iterate, and its proximal gradient point. This algorithm preserves the computational simplicity of classical proximal gradient algorithm [16] where a singular value decomposition in proximal operator is involved. Global convergence is followed directly in the literature.

Authors in $[18,19]$ adopted the SVT algorithm to achieve the completed matrix but by using the power method [20] instead of using PROPACK [21] for computing the singular value decomposition of large and sparse matrix. They showed that accelerating Soft-Impute is indeed possible while still preserving the "sparse plus
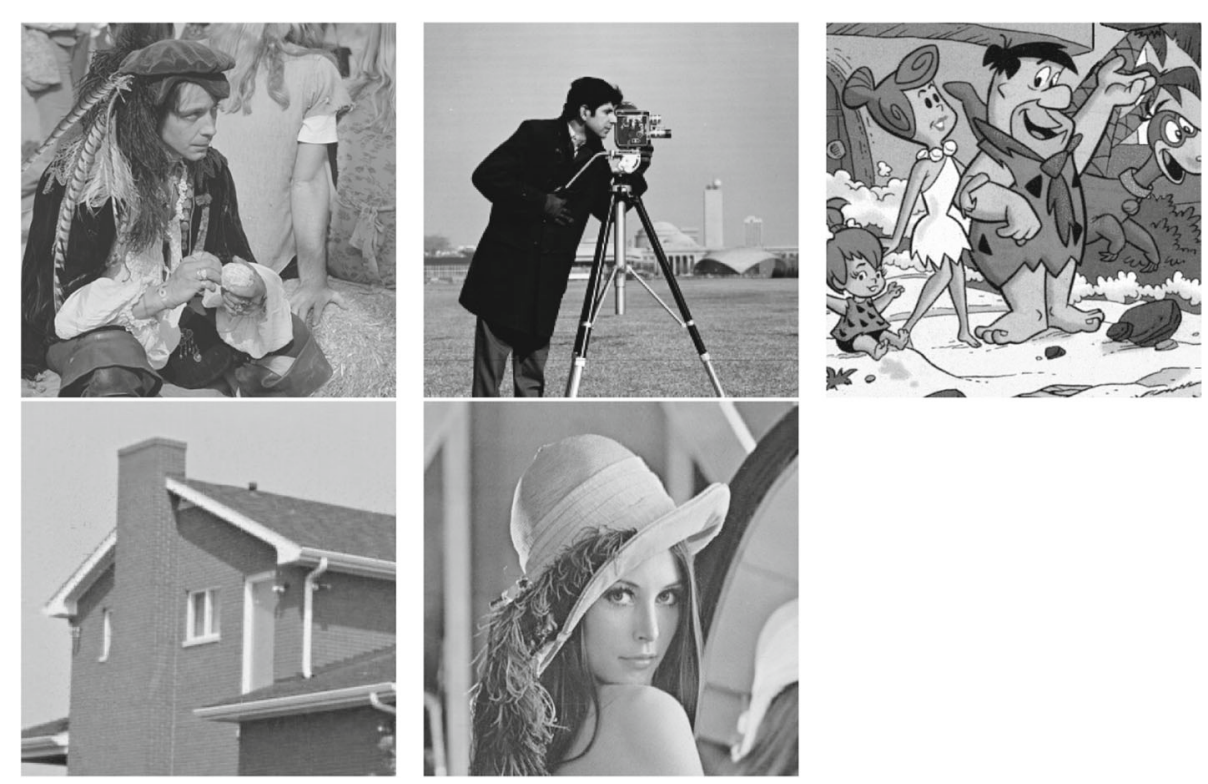

Fig. 1 Benchmark of images. Set of images used in the tests. This is a set of benchmark used as an original image in the tests 
Table 1 PSNR values of the reconstructed images using different ratios

\begin{tabular}{|c|c|c|c|c|}
\hline Image & Method & $\begin{array}{l}15 \% \text { missing } \\
\text { data }\end{array}$ & $\begin{array}{l}25 \% \text { missing } \\
\text { data }\end{array}$ & $\begin{array}{l}35 \% \text { missing } \\
\text { data }\end{array}$ \\
\hline \multirow[t]{7}{*}{ Lena } & FPC & 46.2950 & 45.8888 & 45.6461 \\
\hline & ALM & 48.2790 & 48.2248 & 45.5295 \\
\hline & IALM & 48.2673 & 48.2278 & 46.0819 \\
\hline & PPG & 45.0964 & 41.9579 & 40.2865 \\
\hline & $P G$ & 45.0966 & 41.9582 & 40.2863 \\
\hline & $\begin{array}{l}\text { SVT with } \\
\text { power } \\
\text { method }\end{array}$ & 48.3221 & 48.2745 & 48.2295 \\
\hline & $\begin{array}{l}\text { Proposed } \\
\text { approach }\end{array}$ & 48.9848 & 48.7817 & 48.5952 \\
\hline \multirow[t]{7}{*}{ Cameraman } & FPC & 28.2855 & 32.3433 & 31.7894 \\
\hline & ALM & 36.8075 & 33.9694 & 29.5169 \\
\hline & IALM & 36.7101 & 33.9849 & 29.8495 \\
\hline & PPG & 31.3222 & 27.9425 & 24.3797 \\
\hline & $P G$ & 31.3199 & 27.9458 & 24.3757 \\
\hline & $\begin{array}{l}\text { SVT with } \\
\text { power } \\
\text { method }\end{array}$ & 40.9528 & 40.5182 & 40.0158 \\
\hline & $\begin{array}{l}\text { Proposed } \\
\text { approach }\end{array}$ & 43.3525 & 42.4823 & 40.4596 \\
\hline \multirow[t]{7}{*}{ Flinstones } & FPC & 28.9102 & 37.4826 & 37.2896 \\
\hline & ALM & 42.9923 & 37.3583 & 34.3512 \\
\hline & IALM & 42.8769 & 37.4573 & 34.6774 \\
\hline & PPG & 31.5781 & 29.5989 & 24.3693 \\
\hline & PG & 31.5936 & 29.5887 & 24.3708 \\
\hline & $\begin{array}{l}\text { SVT with } \\
\text { power } \\
\text { method }\end{array}$ & 43.6614 & 43.5614 & 43.2075 \\
\hline & $\begin{array}{l}\text { Proposed } \\
\text { approach }\end{array}$ & 47.8234 & 47.3814 & 44.3920 \\
\hline \multirow[t]{7}{*}{ House } & FPC & 28.6407 & 27.7999 & 27.4826 \\
\hline & ALM & 37.0989 & 36.4579 & 36.3583 \\
\hline & IALM & 36.8952 & 36.5262 & 36.4573 \\
\hline & PPG & 31.5992 & 29.8729 & 29.5998 \\
\hline & $P G$ & 31.6005 & 29.8769 & 29.5701 \\
\hline & $\begin{array}{l}\text { SVT with } \\
\text { power } \\
\text { method }\end{array}$ & 38.1457 & 38.0827 & 37.6494 \\
\hline & $\begin{array}{l}\text { Proposed } \\
\text { approach }\end{array}$ & 39.0707 & 38.1743 & 37.8234 \\
\hline \multirow[t]{7}{*}{ Man } & $\mathrm{FPC}$ & 39.2493 & 38.5864 & 38.1565 \\
\hline & ALM & 41.3640 & 41.2133 & 40.4455 \\
\hline & IALM & 41.2952 & 41.2191 & 40.8616 \\
\hline & PPG & 39.4997 & 39.1895 & 39.3266 \\
\hline & PG & 39.5008 & 39.1889 & 39.3264 \\
\hline & $\begin{array}{l}\text { SVT with } \\
\text { power } \\
\text { method }\end{array}$ & 42.9572 & 42.4158 & 42.0357 \\
\hline & $\begin{array}{l}\text { Proposed } \\
\text { approach }\end{array}$ & 44.3212 & 43.9939 & 43.6724 \\
\hline
\end{tabular}

For each test setting, six results are provided: FPC, ALM, IAM, PPG, PG, SVT with power method, and our proposed model. The best value of the PSNR is the number written in italics on each row low rank" structure. To further reduce the iteration time complexity, instead of computing SVT exactly using PROPACK, they proposed an approximate SVT scheme based on the power method. Though the SVT obtained in each iteration is only approximate, they demonstrated that convergence can still be as fast as performing exact SVT. Hence, the resultant algorithm has low iteration complexity and fast convergence rate. Our objective is to increase the accuracy and the precision of image completion results by adopting unsupervised learning process that takes into account the characteristics of image pixels.

\subsection{Nuclear norm minimization-based collaborating filtering for image reconstruction}

In the problem of collaborating filtering based on nuclear norm minimization, the goal is to predict entries of an unknown matrix based on a subset of its observed entries. For example in a collaborative prediction movie recommendation system, where the rows of the matrix represent users and columns represent movies, the task is to predict ratings that users gave to movies based on their preferences. The prediction of users' preferences over moviesthey have not yet seen-are then based on patterns in the partially observed rating matrix. The setting can be formalized as a matrix completion problem completing entries in a partially observed data matrix.

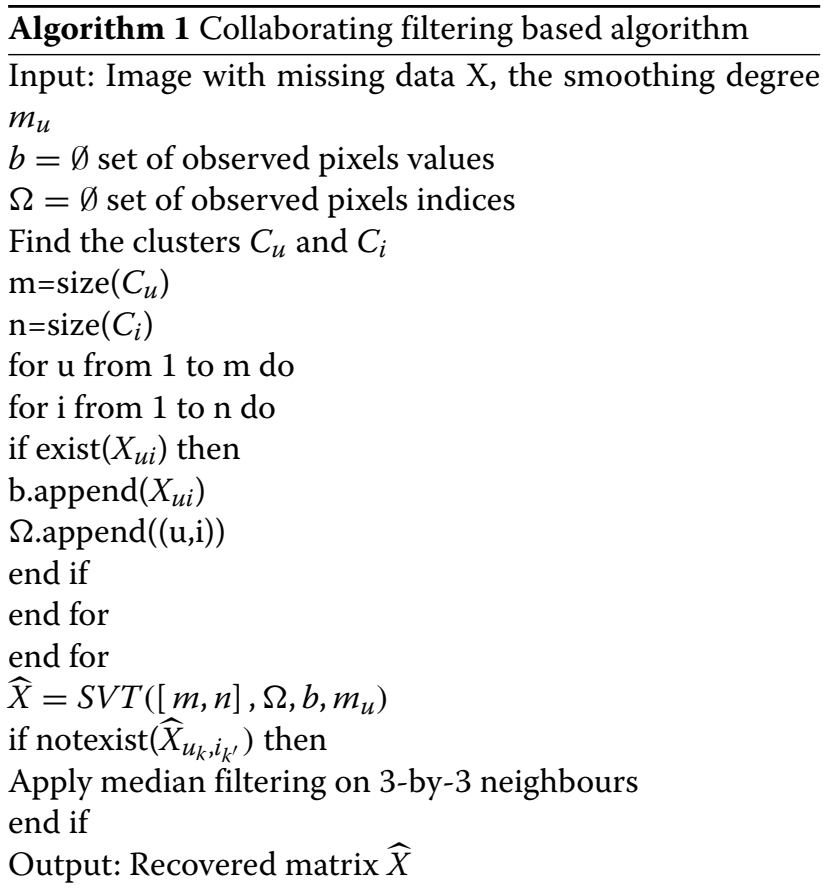

In the same analogy for image completion problem, the collaborating filtering setting aims to predict the pixels missing in the image based on the partially observed 
entries, i.e., pixels in the image. The proposed approach then is based on two main steps:

- Clustering step: uses a learning process to identify pixels' clusters.

- Prediction step: uses a predictive method based on clusters found in the first step to predict the unknown pixels.

Clustering defines the optimal partitioning of a given set of $N$ data points into $K$ subgroups. The points belonging to the same group are as similar as much as possible. However, data points from two different groups share the maximum difference.

The first step of our approach is to perform a data filtering. The learning process starts by applying a principal component analysis (PCA) in the attempt to reduce the number of variables and make the information less redundant. As a result, our data are centered. To detect the pixels' clusters, the process adopts a bi-clustering step founded on prototype-based clustering by using the $K$-means algorithm on the principal component scores, that is, the representation of the data matrix in the principal component space and its correlation matrix.

The second process takes place to predict the missing pixels using the clusters, which performs a new framework for predicting the missing pixels. The clustering phase regroups automatically the pixels of an image into different homogeneous regions. These homogeneous regions usually contain similar objects or part of them. As a result, interesting performance will be achieved in the prediction step.
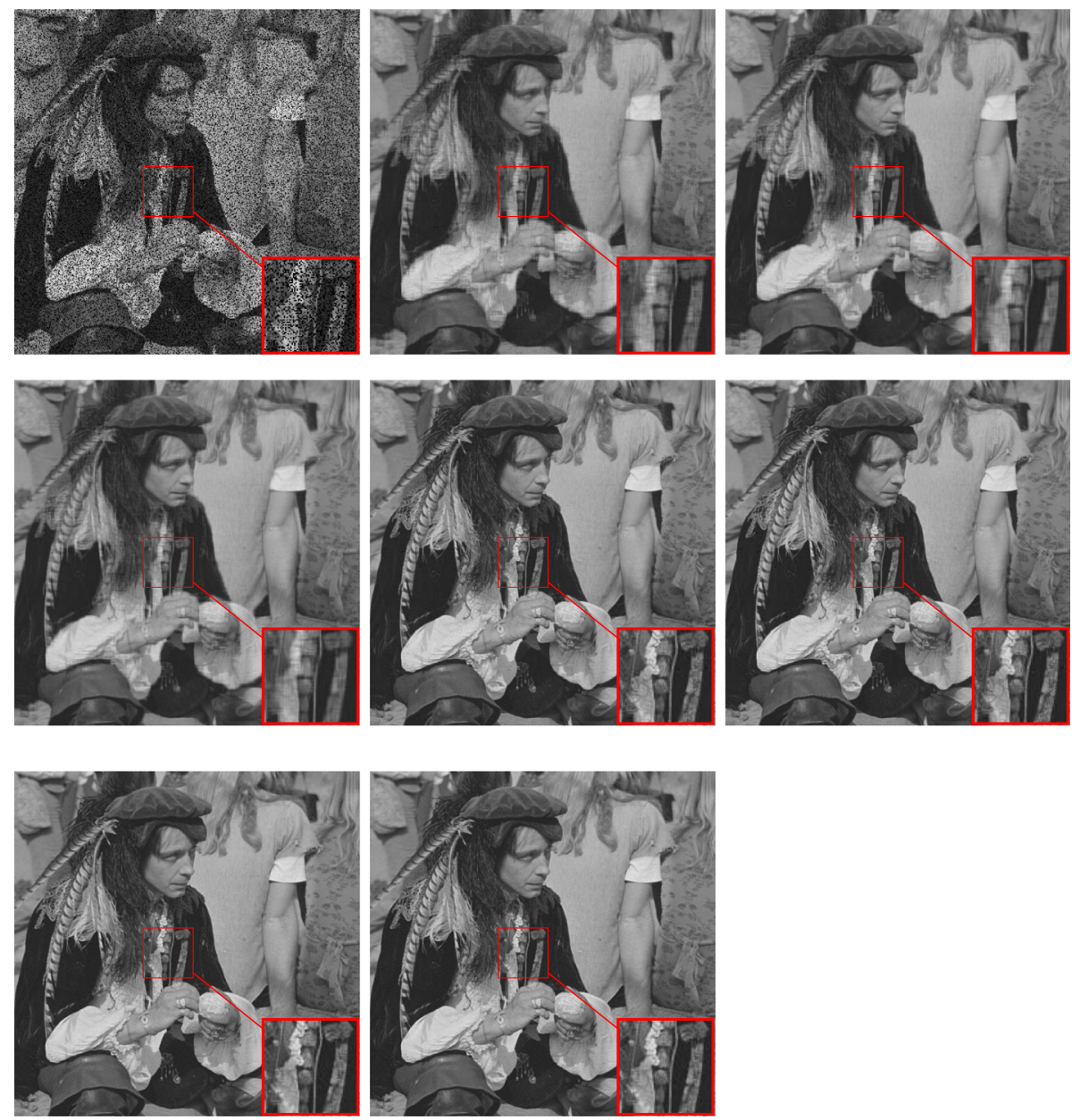

Fig. 2 Results obtained for image "man" using the different methods. From left to right, the order of the images is as follows: simulated image with missing data, recovered image using FPC algorithm, recovered image using ALM algorithm, recovered image using IALM algorithm, recovered image using PPG algorithm, recovered image using PG algorithm, recovered image using SVT with power method, and finally the recovered image using our proposed approach 
For a given point in the image, we identify clusters in which the selected pixel row index, respectively the column index, belongs. The predicted value is the result of singular value thresholding (SVT) algorithm applied on the matrix containing values of pixels existing in the intersection between the two clusters found in step 1 . The adopted algorithm takes as parameters three mandatory elements:

- $\Omega$ the set of locations corresponding to the observed entries.

- $b$ the linear vector which contains the observed elements.

- $m_{u}$ the smoothing degree.

The set of locations corresponding to the observed entries $\Omega$ might be defined in three forms:
- The first one as a sparse matrix where only the elements different of 0 are to take into account.

- The second one as a linear vector that contains the position of the observed elements.

- The third one where $\Omega$ is specified as indices $(i, j)$ with $(i, j) \in \mathbb{N}$.

The application of the proposed algorithm in image completion procures in some cases certain results that are out of range. In this case, we propose to use a median filtering on the predicted pixels. The median filter is often used as a typical pre-processing step to improve the result of later process in signal processing (for example, edge detection on an image). The idea is to use it as a final process to replace each entry (here, entries are the predicted pixels) with the median of neighboring entries, which performs a good result in image reconstruction as shown in the experimental results.
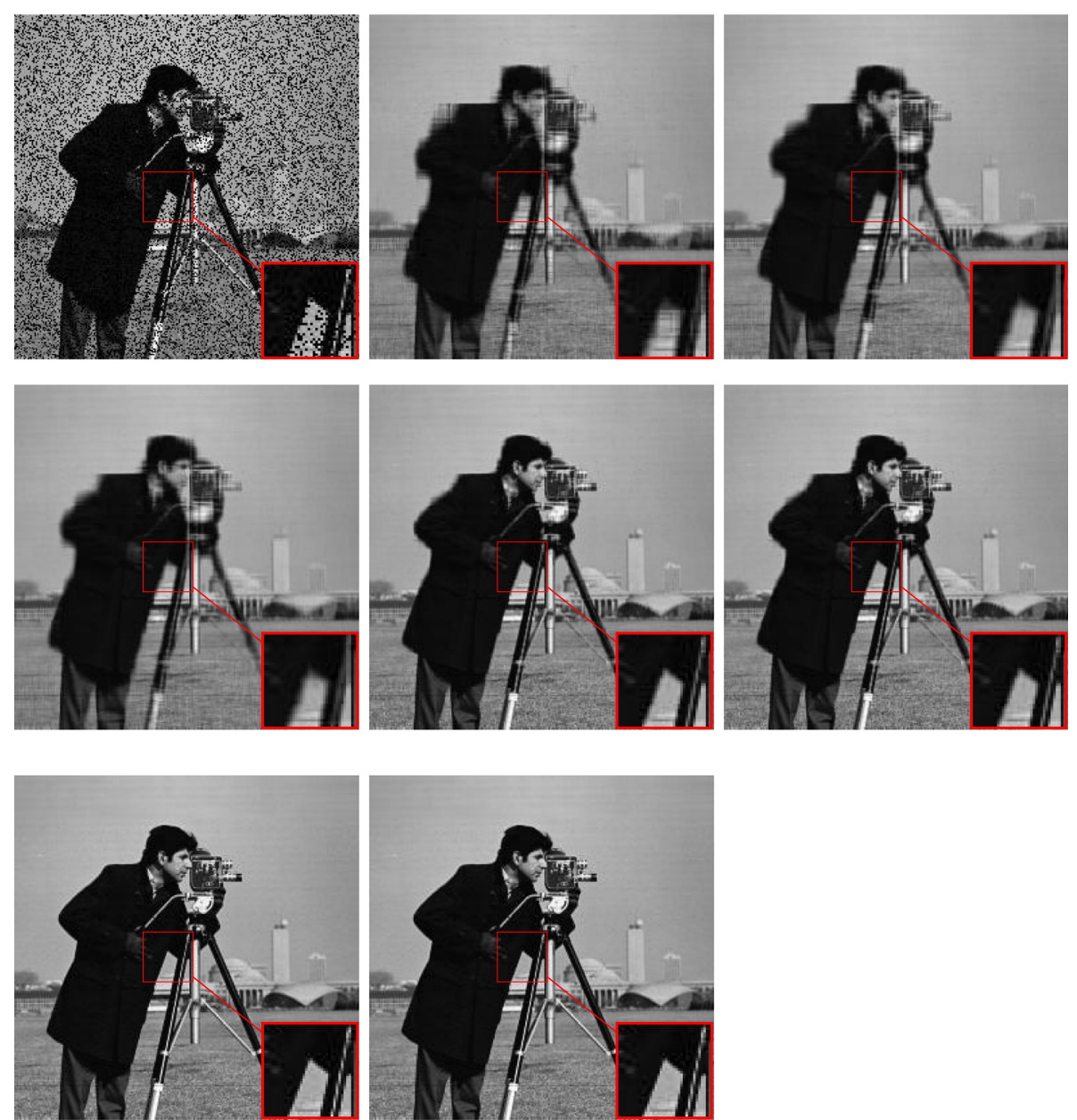

Fig. 3 Results obtained for image "cameraman" using the different methods. From left to right, the order of the images is as follows: simulated image with missing data, recovered image using FPC algorithm, recovered image using ALM algorithm, recovered image using IALM algorithm, recovered image using PPG algorithm, recovered image using PG algorithm, recovered image using SVT with power method, and finally the recovered image using our proposed approach 
The result of our proposed approach is a completed data matrix that contains all the pixels' values. The goal of the proposed approach is to predict the missing pixels in the image matrix. Our learning process detects the partitions of pixels' indices where the predicting process exploits the clusters found to predict the missing value. It works on the assumption that pixels in the same cluster share almost the same characteristics in the image.

\section{Results and discussion}

The proposed approach is compared with several stateof-the-art matrix completion methods including the following: fixed point continuation (FPC) algorithm [22], proximal gradient (PG) algorithm [16, 18, 19], partial proximal gradient (PPG) algorithm [16], augmented Lagrange multiplier (ALM) algorithm [15], and inexact augmented Lagrange multiplier (IALM) algorithm [15].
All these methods need the PROPACK Package [21] for computing the SVD for large and sparse matrix. Our approach was also compared to the method presented in $[18,19]$ that used the power method $[20]$.

The images used are the standards for image processing. We chose a benchmark of five images (Fig. 1) with different gray-level histogram. The computed results are the peak signal-to-noise ratio (PSNR).

We constructed images with arbitrary missing data from the specified benchmark.

In Table 1, the PSNR values are shown for the five different images in Fig. 1 with different choices of the percentage of missing data. The best value of the PSNR is the number written in italics on each row. We can deduce then that our proposed approach is always better than the others, which assures the efficiency of our algorithm.
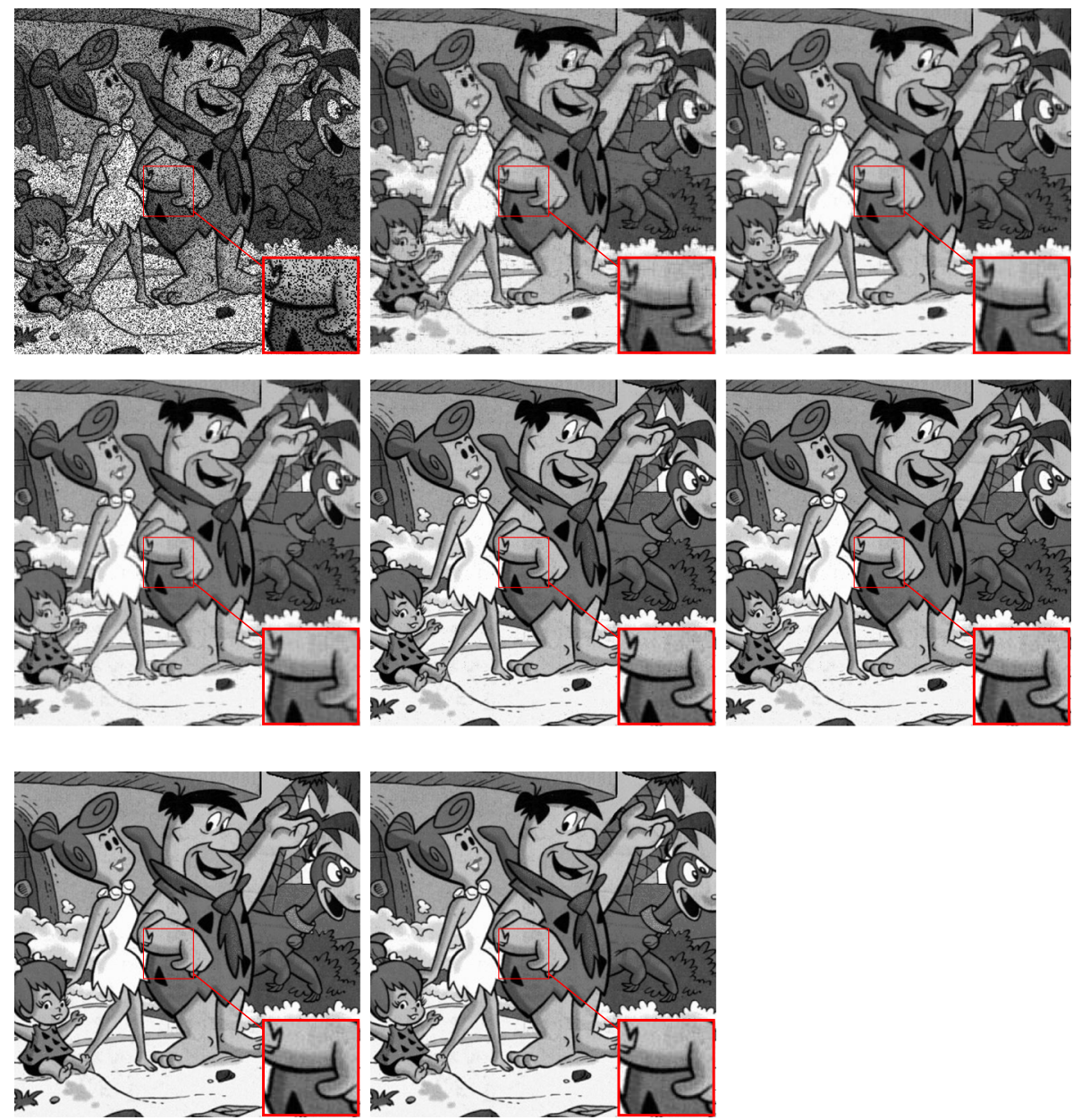

Fig. 4 Results obtained for image "flinstones" using the different methods. From left to right, the order of the images is as follows: simulated image with missing data, recovered image using FPC algorithm, recovered image using ALM algorithm, recovered image using IALM algorithm, recovered image using PPG algorithm, recovered image using PG algorithm, recovered image using SVT with power method, and finally the recovered image using our proposed approach 
In Figs. 2, 3, 4, 5, and 6, we have shown the simulated images compared with the recovered images using our proposed approach, FPC, ALM, IALM, PPG, and PG algorithms. Visually, we can ensure that our approach predicts the missing pixels effectively.

The execution of the main proposed algorithm requires an average of 2 to $10 \mathrm{~min}$ on $2.60 \mathrm{GHz}$ Intel i7 core computer for $256 \times 256$ grayscale images.

The fact that our approach adopts a clustering step to detect the regions with similar pixels allowed us to augment the relevancy and the precision of our SVT-based prediction process. Indeed, when the SVT algorithm is applied on the sub-matrix that contains the pixels of the same cluster, the predicted values procured better PSNR and reconstructed images that are visually consistent more than the SVT algorithm using the power method presented by [18-20]. In addition, the sparcity of the observation matrix made the SVT algorithm the most suitable resolution method for matrix completion problem. Indeed, when recovering the missing image pixels, the FPC, PG, and ALM algorithms procured at their initial phase many iterates that have not a low rank though the optimal solution itself has low rank.

\section{Conclusions}

We propose in this work a new method for image reconstruction from missing data. It is based on two main steps. The first one is a bi-clustering process using $K$-means algorithm to identify pixels' clusters. It is applied on the matrix of PCA scores and its correlation. The second step predicts the missing pixels by applying a matrix completion algorithm on the observations' matrices obtained using the clusters found in step 1 . In each iteration, a matrix of observations is constructed. It contains the
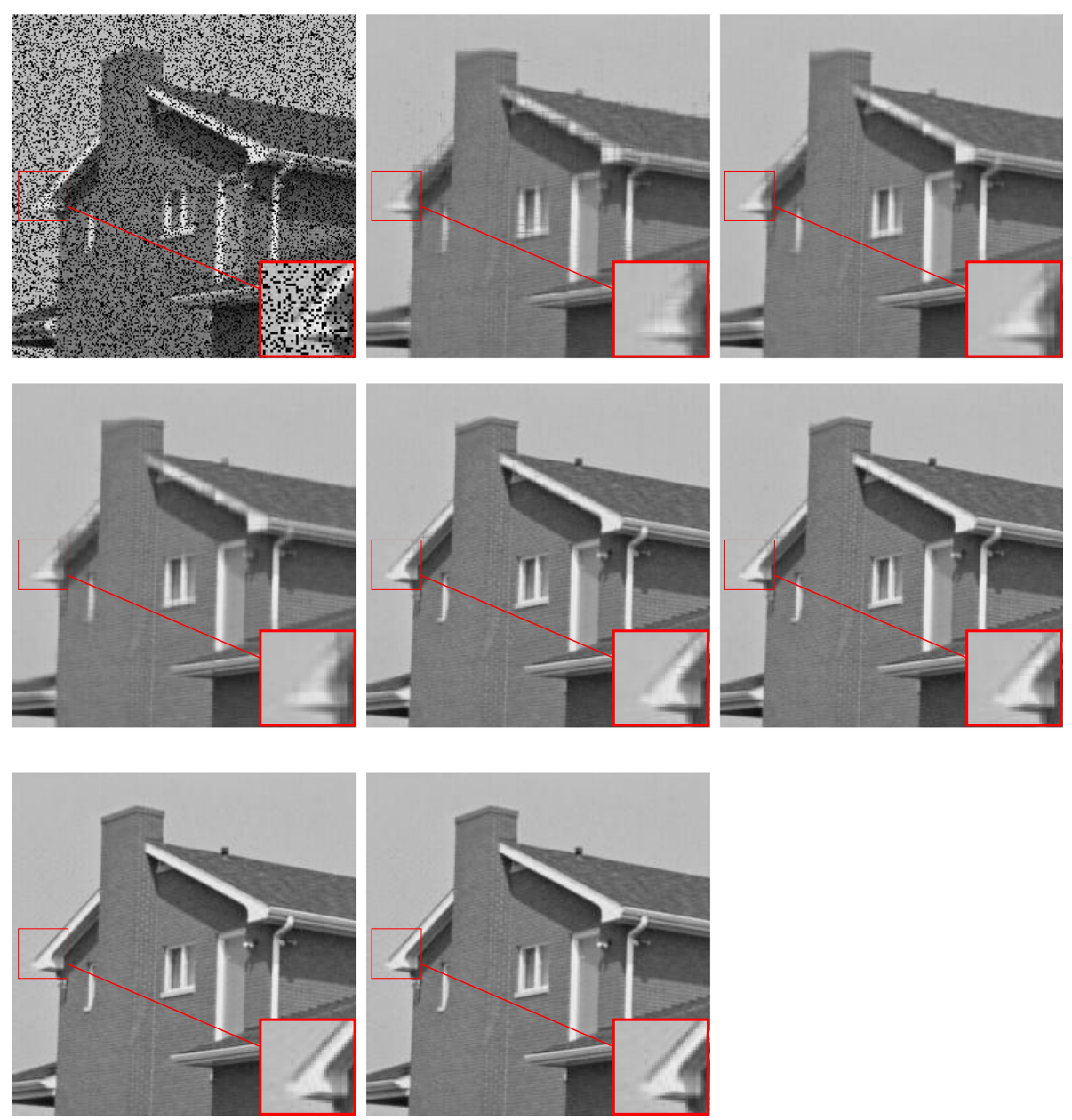

Fig. 5 Results obtained for image "house" using the different methods. From left to right, the order of the images is as follows: simulated image with missing data, recovered image using FPC algorithm, recovered image using ALM algorithm, recovered image using IALM algorithm, recovered image using PPG algoritm, recovered image using PG algorithm, recovered image using SVT with power method, and finally the recovered image using our proposed approach 

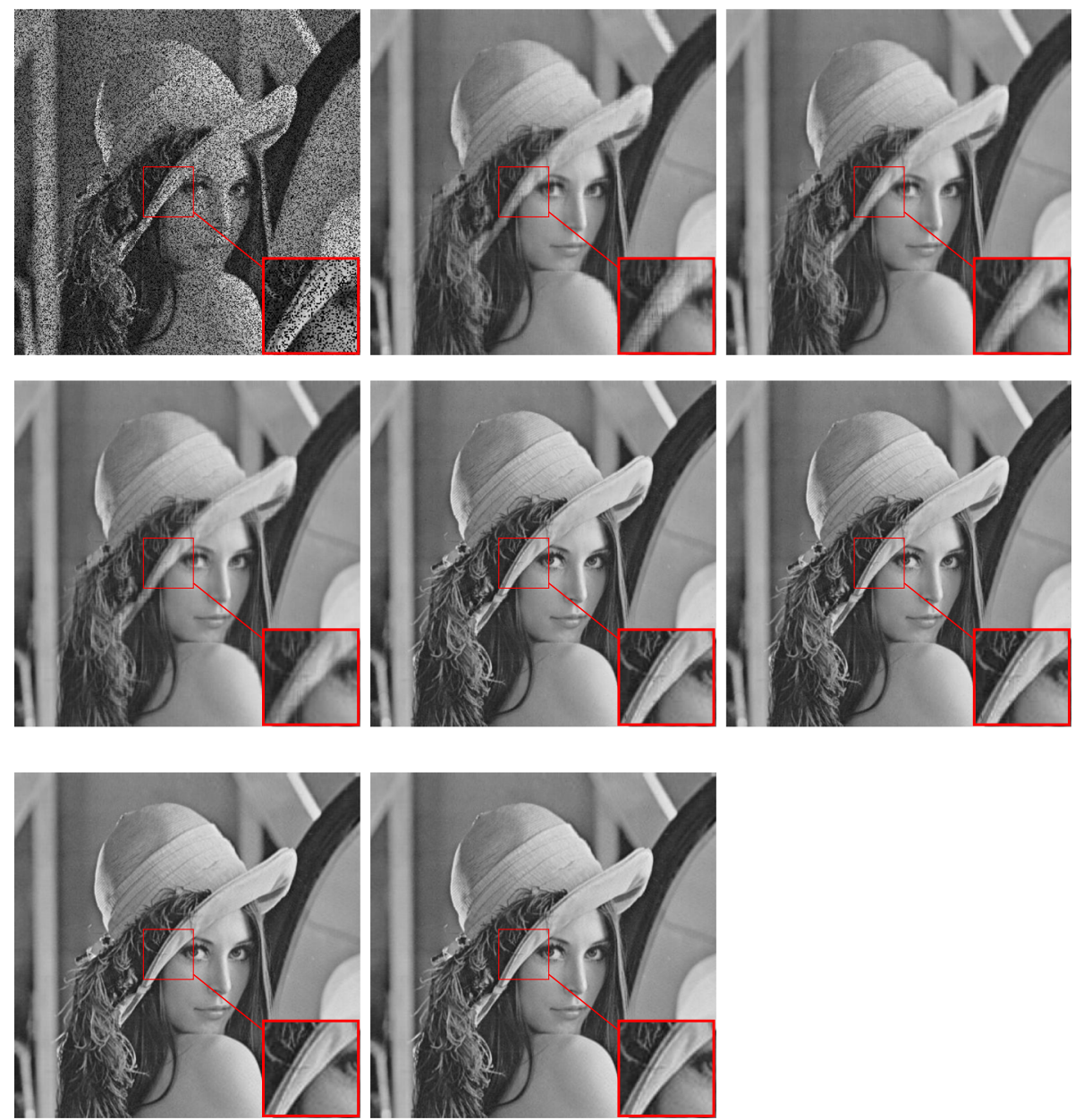

Fig. 6 Results obtained for image "Lena" using the different methods. From left to right, the order of the images is as follows: simulated image with missing data, recovered image using FPC algorithm, recovered image using ALM algorithm, recovered image using IALM algorithm, recovered image using PPG algorithm, recovered image using PG algorithm, recovered image using SVT with power method, and finally the recovered image using our proposed approach

values of pixels that are in the same cluster of the selected missing pixel.

The experimental process is conducted on a benchmark of five standard gray-level images in image processing. The proposed approach is compared visually to different nuclear norm minimization methods for matrix completion and also by measuring the PSNR for different percentages of missing data. Indeed, the proposed approach augments the PSNR of the completion by exploiting the fact that the SVT algorithm is applied per blocks, i.e., on matrices that contain pixels regrouped in the same cluster. A cluster contains eventually pixels that share almost the same characteristics.

\section{Abbreviations}

ALM: Augmented Lagrange multiplier; FPCA: Fixed point continuation algorithm; IALM: Inexact augmented Lagrange multiplier; PCA: Principal component analysis; PG: Proximal gradient; PPG: Partial proximal gradient; PSNR: Peak signal-to-noise ratio; SVT: Singular value thresholding; SVD: Singular value decomposition

\section{Acknowledgements}

Not applicable.

\section{Funding}

Not applicable.

\section{Availability of data and materials}

The benchmark used to demonstrate the effectiveness of the proposed approach is composed of five standard images used for image processing. These images are frequently found in literature and available on the following site: http://www.imageprocessingplace.com/root files V3/image databases.htm

\section{Authors' contributions}

$\mathrm{OB}$ realized the experimental process. For the remaining work, OB, SM, and SR contributed equally to the rest of the work. All authors read and approved the final manuscript.

\section{Authors' information}

Oumayma Banouar, first author, received a PhD degree from the Laboratory of Applied Mathematics and Computer Science, Faculty of Science and Techniques, Cadi Ayyad University, Marrakesh, Morocco.

Souad Mohaoui is a PhD student of the Laboratory of Applied Mathematics and Computer Science, Faculty of Science and Techniques, Cadi Ayyad University, Marrakesh, Morocco. 
Said Raghay received a PhD degree from the Laboratory of Applied Mathematics and Computer Science, Faculty of Science and Techniques, Cadi Ayyad University, Marrakesh, Morocco.

\section{Competing interests}

The authors declare that they have no competing interests.

\section{Publisher's Note}

Springer Nature remains neutral with regard to jurisdictional claims in published maps and institutional affiliations.

Received: 17 January 2018 Accepted: 25 October 2018

Published online: 29 November 2018

\section{References}

1. O. Banouar, S. Raghay, Novel method for users profiles construction through collaborative filtering. IJCSNS. 17, 170-176 (2017)

2. G. Koutrika, Y. loannidis, Personalizing queries based on networks of composite preferences. ACM Trans. Database Syst. 35, 1-50 (2010)

3. Fazel, H. Hindi, S.P. Boyd, in proceedings of the American Control Conference. A rank minimization heuristic with application to minimum order system approximation (IEEE, Arlington, 2001)

4. J. Cai, J.E. Cands, C. Zuowei, A singular value thresholding algorithm for matrix completion. SIAM J. Optim. 20, 1956-1982 (2010)

5. K. Toh, M. Todd, R. Tutuncu, SDPT3, a Matlab software package for semidefinite programming. Optim. Methods Softw. 11, 545-581 (1999)

6. J. Sturm, Using SeDuMi 1.02, a MATLAB toolbox for optimization over symmetric cones. Optim. Methods Softw. 11, 625-653 (1999)

7. O. Klopp, Noisy low-rank matrix completion with general sampling distribution. Bernoulli. 20, 282-303 (2014)

8. J.E. Candès, Y. Plan, in Proceedings of the IEEE, 98. Matrix completion with noise, vol. 98, (2010), pp. 925-936. https://doi.org/10.1109/JPROC.2009. 2035722

9. J.E. Candès, B. Recht, Exact matrix completion via convex optimization. Found. Comput. Math. 9, 717-772 (2009)

10. J.E. Candès, X. Li, Y. Ma, J. Wright, Robust principal component analysis. JACM. 58, 37 (2011). https://doi.org/10.1145/1970392.1970395

11. O. Banouar, S. Raghay, User profile construction for personalized access to multiple data sources through matrix completion method. IJCSNS. 16, 51-57 (2016)

12. S. Ma, D. Goldfarb, L. Chen, Fixed point and Bregman iterative methods for matrix rank minimization. Optim. Control. 128, 321-353 (2011)

13. P.L. Combettes, V.R. Wajs, Signal recovery by proximal forward-backward splitting. Multiscale Model. Simul. 4, 1168-1200 (2005)

14. S. Osher, M. Burger, D. Goldfarb, J. Xu, W. Yin, An iterative regularization method for total variation-based image restoration. Multiscale Model. Simul. 4, 460-489 (2005)

15. Z. Lin, M. Chen, L. Wu, Y. Ma, The augmented Lagrange multiplier method for exact recovery of corrupted low-rank matrices (2010). UIUC Technical Report UILU ENG-09-2215 arXiv:1009.5055 [math.OC]

16. Z. Lin, A. Ganesh, J. Wright, L. Wu, M. Chen, Y. Ma, in Intl. Workshop on Comp. Adv. in Multi-Sensor Adapt. Processing, Aruba, Dutch Antilles. Fast convex optimization algorithms for exact recovery of a corrupted low-rank matrix. UIUC Technical Report UILU-ENG-09-2214, (2009)

17. Q. Wang, W. Cao, Z. Jin, Two-step proximal gradient algorithm for low-rank matrix completion. Stat. Optim. Inf. Comput. 4(2), 201-210 (2016)

18. Q. Yao, J.T. Kwok, in Proc. of the Int. Joint Conf. on Art. Intel. Accelerated inexact soft impute for fast large scale matrix completion and tensor completion (AAAI Press, Buenos Aires, 2015)

19. Q. Yao, J.T. Kwok, Accelerated inexact soft impute for fast large scale matrix completion and Tensor completion. IEEE Trans. Knowl. Data Eng. (2017). arXiv:1703.05487v2 [cs.NA]

20. N. Halko, P.G. Martinsson, J.A. Tropp, Finding structure with randomness: probabilistic algorithms for constructing approximate matrix decompositions. SIAM. Rev. 53(2), 217288 (2011)

21. K.C. Toh, S. Yun, An accelerated proximal gradient algorithm for nuclear norm regularized linear least squares problems. Pac. J. Optim. 6, 615-640 (2010)

22. E.T. Hale, W. Yin, Y. Zhang, Fixed-point continuation for I1-minimization: methodology and convergence. SIAM J. Optim. 19, 1107-1130 (2008)

\section{Submit your manuscript to a SpringerOpen ${ }^{\circ}$ journal and benefit from:}

- Convenient online submission

Rigorous peer review

- Open access: articles freely available online

- High visibility within the field

- Retaining the copyright to your article

Submit your next manuscript at $>$ springeropen.com 